\title{
Adam22 Is a Major Neuronal Receptor for Lgi4-Mediated Schwann Cell Signaling
}

\author{
Ekim Özkaynak, ${ }^{1 \star}$ Gina Abello, ${ }^{1 \star}$ Martine Jaegle, ${ }^{1}$ Laura van Berge, ${ }^{1}$ Diana Hamer, ${ }^{1}$ Linde Kegel, ${ }^{1}$ Siska Driegen, ${ }^{1}$ \\ Koji Sagane, ${ }^{2}$ John R. Bermingham Jr, ${ }^{3}$ and Dies Meijer ${ }^{1}$ \\ ${ }^{1}$ Department of Cell Biology and Genetics, Erasmus University Medical Center, 3015 GE Rotterdam, The Netherlands, ${ }^{2}$ Tsukuba Research Laboratories, \\ Eisai Co. Ltd., Tokodai 5-1-3, Tsukuba, Ibaraki 300-2635, Japan, and ${ }^{3}$ McLaughlin Research Institute, Great Falls, Montana 59405
}

The segregation and myelination of axons in the developing PNS, results from a complex series of cellular and molecular interactions between Schwann cells and axons. Previously we identified the Lgi4 gene (leucine-rich glioma-inactivated4) as an important regulator of myelination in the PNS, and its dysfunction results in arthrogryposis as observed in claw paw mice. Lgi4 is a secreted protein and a member of a small family of proteins that are predominantly expressed in the nervous system. Their mechanism of action is unknown but may involve binding to members of the Adam (A disintegrin and metalloprotease) family of transmembrane proteins, in particular Adam22. We found that Lgi4 and Adam22 are both expressed in Schwann cells as well as in sensory neurons and that Lgi4 binds directly to Adam 22 without a requirement for additional membrane associated factors. To determine whether Lgi4-Adam22 function involves a paracrine and/or an autocrine mechanism of action we performed heterotypic Schwann cell sensory neuron cultures and cell typespecific ablation of Lgi4 and Adam22 in mice. We show that Schwann cells are the principal cellular source of Lgi4 in the developing nerve and that Adam 22 is required on axons. Our results thus reveal a novel paracrine signaling axis in peripheral nerve myelination in which Schwann cell secreted Lgi4 functions through binding of axonal Adam22 to drive the differentiation of Schwann cells.

\section{Introduction}

The myelin sheath is a highly ordered lamellar structure that surrounds a large fraction of nerve fibers in the vertebrate nervous system. Myelinated nerve fibers conduct nerve impulses with a speed that is approximately two orders of magnitude faster than unmyelinated fibers of similar diameter. Dedicated neuroglial cells, oligodendrocytes in the CNS and Schwann cells in the PNS, produce myelin. The importance of the myelin sheath for the normal function of the nervous system is underscored by the large range of neurological diseases associated with its dysfunction.

The structural and functional maturation of myelinated nerve fibers in the PNS is governed by a temporally and spatially controlled series of molecular interactions between the axon and the Schwann cell (Jessen and Mirsky, 2005). For example, cell adhesion molecules of the nectin-like family are important for proper

Received Dec. 20, 2009; revised Jan. 25, 2010; accepted Feb. 8, 2010.

This work was supported by grants from the Dutch government; Netherlands Organization for Scientific Research VICI Grant 918.66.616 (D.M.), BSIK programme 03038, 'Stem cells in development and disease' (D.M.), the European Community's Seventh Framework Programme (FP7/2007-2013) under Grant Agreement No. HEALTH-F2-2008201535 (D.M.), National Institute of Neurological Disorders and Stroke Grant R01NS40751 (J.R.B.), and Muscular Dystrophy Association Grant 4147 (J.R.B.). G.A. is a recipient of a European Union FP7 Marie Curie Fellowship (PIEF-GA-2009-236638). We thank Tom de Vries Lentsch for help in preparing the figures, Ivo Spiegel and Elior Peles (Weizmann Institute, Rehovot, Israel) for Fc-fusion expression cassettes, Carla Taveggia (San Raffaele, Milan, Italy) for ErbB2-Fc and ErbB3-Fc vectors and expert advice, and Elaine Dzierzak for critically reading the manuscript.

*E.Ö. and G.A. contributed equally to this work.

Correspondence should be addressed to Dr. Dies N. Meijer, Department of Cell Biology and Genetics, Erasmus University Medical Center, Dr Molewaterplein 50, 3015 GE Rotterdam, The Netherlands. E-mail: d.meijer@erasmusmc.nl.

DOI:10.1523/JNEUROSCI.6287-09.2010

Copyright $\odot 2010$ the authors $\quad 0270-6474 / 10 / 303857-08 \$ 15.00 / 0$
Schwann cell-axon interactions during myelination and along the internode of the structurally mature myelin sheath (Maurel et al., 2007; Spiegel et al., 2007). Furthermore, engagement of axonally derived neuregulin-1 ( $\mathrm{Nrg} 1$ ) with ErbB2/3 tyrosine-kinase receptors on Schwann cells mediates Schwann cell proliferation and survival, and myelination of axons (Nave and Salzer, 2006; Birchmeier and Nave, 2008). Other ligands for tyrosine kinase receptors also modulate myelination, including platelet-derived growth factor and insulin-like growth factor-1 (Ogata et al., 2006) and the neurotrophins (Chan et al., 2001). Additionally, the G-protein coupled receptor gpr126 is an orphan receptor that is Schwann cell-autonomously required to regulate expression of the transcription factor Oct6 and induce myelination (Monk et al., 2009).

Recently, a mutant form of Lgi4 has been shown to underlie the congenital hypomyelinating phenotype of claw paw mice (Henry et al., 1991; Bermingham et al., 2006). Lgi4 is a secreted molecule that belongs to a small family of proteins predominantly expressed in the nervous system. In claw paw mice the Lgi4 mutant protein is not secreted (Bermingham et al., 2006). Thus, the Lgi4 protein is implicated as a positive regulator of myelin formation in the PNS.

Previous studies suggest that proteins of the ADAM (A disintegrin and metalloproteinase) family (Seals and Courtneidge, 2003; Yang et al., 2006) are receptors for Lgi proteins. Lgil and Adam22 coprecipitate from brain membrane preparations (Fukata et al., 2006; Schulte et al., 2006) and Lgil and Lgi4 both bind to cells that ectopically express Adam 22 or Adam 23 (Fukata et al., 2006; Sagane et al., 2008). Strikingly, deletion of Adam22 presents a peripheral nerve phenotype similar to that of claw paw 
animals (Sagane et al., 2005) suggesting that Adam22 is a receptor for Lgi4 in the developing nerve. However, it is unknown whether Lgi4 interacts directly with Adam22 and whether the Lgi4Adam22 ligand receptor pair functions through an autocrine or (juxta)paracrine mechanism of action. Here we show that Lgi4 binds directly to the ectodomain of Adam22. Lgi4 binding depends on an intact Adam22 disintegrin domain but is independent of additional membrane associated factors. Using cell typespecific deletion of Lgi4 or Adam 22 and heterotypic sensory neuron-Schwann cell cultures we show that Schwann cells are the principal source of Lgi4 and require binding to axonal Adam 22 to drive myelin formation.

\section{Materials and Methods \\ Mice}

The neo allele of Adam22 (Adam22 $2^{\text {neo }}$ ), in which a TK-neo cassette is inserted into exon 8, has been described before (Sagane et al., 2005). Conditional alleles for Adam 22 and Lgi4 were generated through homologous recombination in ES cells using standard techniques (Jaegle et al., 2003). To create a conditional Lgi4-null allele we introduced LoxP sites into a nonconserved CA repeat $5^{\prime}$ to the Lgi4 translation start site, and a second LoxP site, along with a PGK-neomycin cassette that was flanked with frt sites, into intron 3 through homologous recombination in ES cells. We established mouse lines from two independently targeted ES cell lines; these mice were phenotypically indistinguishable. The PGK-neo cassette was removed by crossing the mice with the ROSA26-Frt recombinase deleter mouse (Farley et al., 2000). Offspring of this cross was used to establish the Lgi4 conditional knock out (Lgi4 ${ }^{\text {Lox }}$ ) mouse line used here. Crossing these mice with Cre recombinase drivers such as DhhCre and Wnt1Cre, results in the tissue-specific deletion of exon 1-3, including the Lgi4 translation start site, signal sequence and N-terminal leucine-rich repeat, thereby generating a null allele (see Fig. $1 A$ ). To create a conditional null allele for Adam $22\left(\operatorname{Adam} 22^{\text {Lox }}\right)$, we introduced LoxP sequences around exon1, along with a TK-neo cassette that was flanked by Frt sites, through homologous recombination in ES cells. Correctly targeted ES cells were used to generate a mouse carrying the targeted allele containing the TK-neo selection cassette. The TK-neo cassette was removed by crossing the mice with the ROSA26-Frt recombinase deleter mouse (Farley et al., 2000). Offspring of this cross was used to establish the Adam 22 conditional knock out (Adam $22^{\text {Lox }}$ ) mouse line. Deletion of exon1 by Cre recombinase results in the generation of the Adam22-null $\left(\operatorname{Adam} 22^{\Delta 1}\right.$ ) allele (see Fig. 5A). All animal experiments were performed in compliance with the relevant laws and institutional guidelines and were approved by an independent committee (DEC) on the ethical use of experimental animals.

\section{Light microscopy}

Mice were deeply anesthetized with sodium pentobarbital and transcardially perfused with PBS followed by $4 \%$ paraformaldehyde/1\% gluteraldehyde in $0.1 \mathrm{~m}$ phosphate buffer $\mathrm{pH}$ 7.2. Sciatic nerves, spinal cords and DRGs were dissected and placed in the same fixative at $4^{\circ} \mathrm{C}$ for at least $16 \mathrm{~h}$. Tissues were washed with $0.1 \mathrm{M}$ phosphate buffer and osmicated overnight at $4^{\circ} \mathrm{C}$ in $1 \%$ osmium tetroxide/ferricyanide in phosphate buffer. Tissues were embedded in plastic resin and $1 \mu \mathrm{m}$ sections were cut, mounted and stained with either paraphenylenediamine (PPD) or toluidine blue, as described previously (Estable-Puig et al., 1965; Jaegle et al., 2003). Sections were examined using an Olympus BX40 microscope and pictures were collected using a ColorviewIIIu camera.

\section{Immunofluorescence}

Mice were killed at postnatal day 12 (P12) and sciatic nerves were dissected and fixed in $4 \%$ PFA in PBS overnight at $4^{\circ} \mathrm{C}$ and embedded in paraffin. Six micron sections were rehydrated and washed in PBS and $\mathrm{PBS} / 0.1 \%$ Tween 20. Antigen retrieval was performed with $15 \mathrm{~mm} \mathrm{Na}_{3}$ Citrate Buffer containing $0.05 \%$ Tween 20 at $80-90^{\circ} \mathrm{C}$ for $20-30 \mathrm{~min}$ in a water bath. Sections were blocked for at least $1 \mathrm{~h}$ at room temperature with PBS, $1 \%$ BSA, $0.2 \mathrm{~mm}$ glycine, $0.05 \%$ Tween 20 , and $0.5 \%$ Normal Goat Serum (Dako) or Normal Donkey Serum (Sigma) depending on the secondary antibody used. Primary antibodies were diluted in blocking solution, and sections were incubated overnight at $4^{\circ} \mathrm{C}$. Secondary antibodies were incubated in block solution for $2 \mathrm{~h}$ at room temperature. After washing and rinsing in distilled water, sections were dried, and mounted with polyvinyl alcohol 4-88 containing DABCO anti-fading agent and DAPI for DNA staining. Samples were examined under a Zeiss AxioImager.Z1 microscope and images were captured with an AxioCam $\mathrm{MRm}$ digital camera, and analyzed with AxioVision software. Brightness and contrast levels were adjusted in Adobe PhotoShop.

\section{Binding assays}

Lgi2, Lgi3 or Lgi4 conditioned media (CM) were incubated with antibodies against V5-tag (Lgi2 and Lgi3) or Myc-tag (Lgi4) for $45 \mathrm{~min}$ at room temperature. CM were then added to transfected cells and incubated at $37^{\circ} \mathrm{C} / 5 \% \mathrm{CO}_{2}$ for $45 \mathrm{~min}$. Cells were washed twice with PBS, fixed with 4\% PFA in PBS for at least 15 min, then washed twice with PBS, once with PBS/0.05\% Triton X-100, and blocked for at least $20 \mathrm{~min}$ at room temperature. Block contained 1\% BSA, $0.2 \mathrm{~mm}$ glycine, $0.01 \%$ Triton X-100, and 0.5\% Normal Goat Serum (Dako). Secondary antibody incubation was done at room temperature for at least $45 \mathrm{~min}$.

\section{Sciatic nerve tissue extraction and Western blotting}

Nerves were dissected from killed animals at P12 and frozen on dry ice. Tissues were extracted in reducing sample buffer by sonicating on ice, with $4 \mathrm{on} / \mathrm{off}$ cycles of $1 \mathrm{~min}$. Proteins were separated by SDS-PAGE (12.5\% gel) and blotted onto Immobilon-P PVDF membranes (Millipore). Blots were probed with different antibodies exactly as described before (Jaegle et al., 2003).

\section{Antibodies}

Primary antibodies. Primary antibodies were from the following sources: Mouse-anti-P0 (Archelos et al., 1993) [immunofluorescence (IF), 1:500; Western blot (WB), 1:1000], rabbit-anti-Oct6 (Jaegle et al., 2003) (IF, 1:200; WB, 1:500), rabbit-anti-Krox20 (Ghazvini et al., 2002) (IF, 1:200; WB, 1:500), mouse-anti-neurofilament M (IF, 1:300) (The 2H3 monoclonal antibody developed by Jessell and colleagues was obtained from the Developmental Studies Hybridoma Bank, developed under the auspices of the National Institute of Child Health and Human Development and maintained by the Department of Biology, University of Iowa, Iowa City, IA), rat-anti-myelin basic protein (Millipore; IF, 1:300), goat-antiChAT (Millipore; IF, 1:200), mouse-anti-myc 9E10 (clone 9E10, 0.4 mg/ $\mathrm{ml}$, Roche; IF, 1:300), mouse-anti-V5 (custom-made monoclonal antibody; IF, 1:50), rabbit-anti-phospho AKT (Cell Signaling Technology; WB, 1:1000), rabbit-anti-Erk (Cell Signaling Technology; WB, 1:1000), mouse-anti-acetylated tubulin (Sigma; WB, 1:1000).

Secondary antibodies. The following secondary antibodies were used. Alexa594-conjugated goat-anti-mouse and Alexa488-conjugated oat-antirabbit (Invitrogen; used at 1:300 for IF), Cy2-conjugated donkey-antimouse (IF, 1:100), AffiniPure DyLight594-conjugated donkey-anti-rat and AffiniPure DyLight594-conjugated donkey-anti-goat (Jackson ImmunoResearch; IF: 1:500), HRP-goat-anti-mouse, AP-goat-anti-mouse, and HRP-goat-anti-rabbit (Dako; WB, 1:5000), goat-anti-human-HRP (GE Healthcare; WB, 1:5000).

\section{Cloning}

Lgi2, 3, 4 and Adam22 cDNAs were amplified from E13.5 mouse DRG cDNA using Phusion polymerase (Finnzymes), cloned into Zero-Blunt TOPO vector (Invitrogen) and sequenced. cDNAs were generated using the SuperScript II (Invitrogen) reverse transcription system, on total RNA isolated by RNA-Bee reagent (Tel-Test, Inc). Adam 11 and Adam 23 were amplified from IMAGE cDNA clones. V5-His (Lgi2, 3) or myc-His (Lgi4) tagged Lgi proteins were obtained by recloning into pcDNA3.1based expression cassettes. Full-length Adam-GFP-His fusion proteins and Ectodomain Adam-Fc fusion proteins were obtained by recloning into CMV-based expression cassettes [G20 isoform for Adam22 and $\alpha$ isoform for Adam23 were used (Sun et al., 2004; Sagane et al., 2005)].

\section{Cell culture and immunoprecipitation assays}

All cell culture reagents were from Lonza, and all cultures dishes were from Greiner Bio-One. CHO and HeLa cells were grown in DMEM, 10\% 


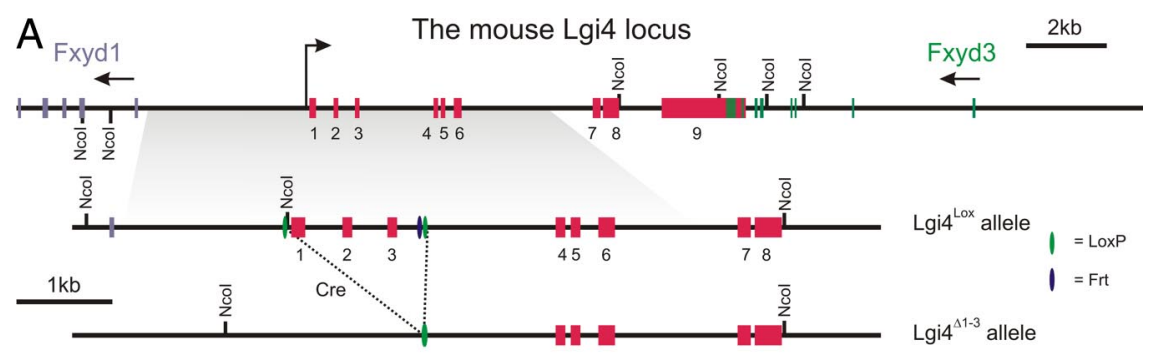

B

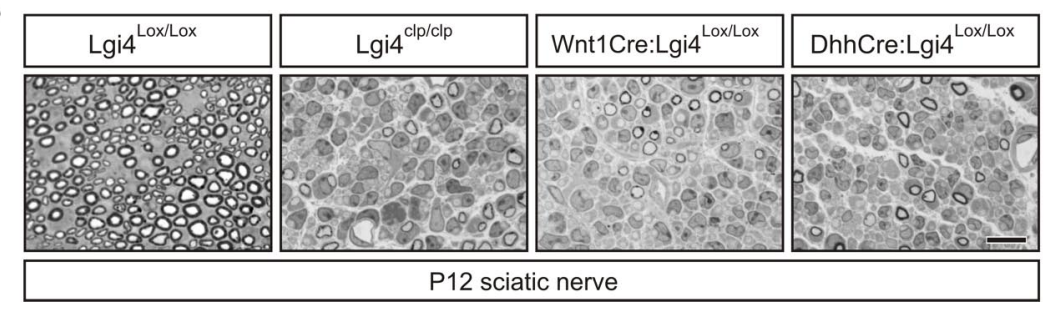

Figure 1. Schwann cell-specific deletion of Lgi4 results in a congenital hypomyelinating phenotype in the peripheral nervous system. $A$, Schematic depiction of the $L g i 4$ gene, the conditional allele $\left(L g i 4^{L o x}\right)$, and the null allele $\left(L g i 4^{\Delta 1-3}\right)$ that results from the action of Cre recombinase. The $L$ gi 4 gene consists of 9 exons spanning $\sim 12 \mathrm{~kb}$ of genomic sequences on mouse chromosome 7 . The gene is located between the Fxyd7 and Fxyd3 genes. The Lgi4 and Fxyd3 3 'ends overlap. The arrows indicate the direction of transcription. $\boldsymbol{B}$, Cross sections of plastic embedded nerves derived from animals homozygous for the conditional Lgi4 allele $\left(\right.$ Lgit $\left.{ }^{L 0 x / L x}\right)$ at P12, show a pattern of myelin figures that is comparable to wild type (see Fig. 2A). Deletion of the Lgi4 gene in

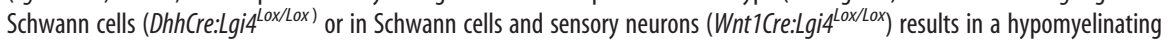
phenotype with few myelinated axons, similar to that observed in claw paw $\left(\mathrm{Lgi}_{\mathrm{i}}^{\mathrm{cl} / \mathrm{c} / \mathrm{cl} p}\right)$ animals. Sections are stained with PPD to accentuate the myelin sheath, which appears as a dark ring. Scale bar represents $10 \mu \mathrm{m}$.

FCS, penicillin and streptomycin (PS), and HEK 293T cells were grown in DMEM/F10, 10\% FCS, PS. HeLa, or HEK 293T cells were transiently transfected using Lipofectamine reagent (Invitrogen) or the calcium phosphate transfection method. For immunoprecipitation assays, media conditioned for $6 \mathrm{~d}$ were collected and filtered. The conditioned medium was buffered with $0.1 \mathrm{~m}$ Tris $\mathrm{pH} 8$ and Adam22-ED-Fc proteins were bound to protein $\mathrm{A}$ beads (RepliGen) overnight at $4^{\circ} \mathrm{C}$. Adam22-ED-Fc beads were washed three times with PBS/0.1\% Triton X-100 and then added to Lgi4 conditioned medium and incubated overnight at $4^{\circ} \mathrm{C}$. After washing, beads were resuspended in reducing sample buffer. Bound proteins were separated by SDS-PAGE (10\% resolving gel) and analyzed by Western blotting.

\section{Rat Schwann cell and DRG cultures}

Primary rat Schwann cell cultures were set up essentially as described before (Brockes et al., 1979) with slight modifications.

DRGs were isolated aseptically from E13.5 mouse embryos. After dissociation by trypsinization and trituration, the cells were plated onto 18 $\mathrm{mm}$ poly-L-lysine (Sigma)/collagen-coated coverslips (Thermo Scientific), and maintained in MEM (Invitrogen), 3\% FCS, $100 \mathrm{ng} / \mathrm{ml} \mathrm{NGF,} \mathrm{PS}$ (M1 Medium). The medium was refreshed every other day. To obtain neuron-only cultures, the cells were treated with $10 \mu \mathrm{M}$ FUDR (Sigma) in M1 medium for $2 \mathrm{~d}$. Two cycles of FUDR treatment ( $2 \mathrm{~d}$ with FUDR, $2 \mathrm{~d}$ without FUDR) was usually sufficient to obtain neuron-only cultures. Rat Schwann cells were starved of neuregulin in DMEM, 10\% FCS, PS for $3 \mathrm{~d}$ before seeding $2 \times 10^{5}$ cells in M1 medium onto neuron-only cultures. The Schwann cells were allowed to associate with the neurons for an additional $5 \mathrm{~d}$, and then myelination was induced by addition of ascorbic acid to $50 \mu \mathrm{g} / \mathrm{ml}$ (Sigma). Cultures were maintained in M1 medium containing $50 \mu \mathrm{g} / \mathrm{ml}$ ascorbic acid and 10\% FCS for 2 weeks and subsequently analyzed for the presence of myelin.

\section{Results}

\section{Cellular source of Lgi4 in the developing nerve}

Schwann cells are a likely source of Lgi4 as siRNA inhibition of Lgi4 expression in Schwann cells reduces myelin formation in neuron-Schwann cell coculture experiments (Bermingham et al., 2006). However, both Schwann cells and sensory neurons express

\section{Lgi4 receptors}

Lgi4 mRNA (Bermingham et al., 2006) (supplemental Fig. 1, available at www. jneurosci.org as supplemental material), and nerve transplantation experiments have suggested both Schwann cell autonomous and possibly nonautonomous functions of Lgi4 (Darbas et al., 2004). Whether Lgi4 is expressed in mesenchymal derivatives such as the perineural cells or endothelial cells and pericytes, is unknown. To address the cell-autonomous function of Lgi4 in the PNS, we generated mice that carry a conditional null allele of Lgi4 (Fig. 1A). Lgi4 was specifically deleted during embryonic development in Schwann cells, sensory neurons (but not motor neurons) and endoneurial fibroblasts, using a Wnt1Cre transgene (Danielian et al., 1998; Joseph et al., 2004). Microscopic examination of cross sections of sciatic nerves of Wnt1Cre:Lgi4 ${ }^{\text {Lox/Lox }}$ animals at postnatal day 12 (P12) reveals a hypomyelination phenotype that is indistinguishable from that observed in claw paw (Lgi4 ${ }^{\text {clp } / c l p}$ ) nerves (Fig. $1 B$ ). To further define the cellular source of Lgi4, we examined nerves of animals in which Lgi4 is deleted during embryonic development in Schwann cells and endoneurial fibroblasts only, using a DhhCre transgene (Jaegle et al., 2003). At P12, DhhCre:Lgi4 ${ }^{\text {Lox/Lox }}$ animals present with a hypomyelination phenotype that is identical to that of claw paw and Wnt1Cre:Lgi4 ${ }^{\text {Lox/Lox }}$ mice (Fig. 1B). Taken together, these results demonstrate that Schwann cells are the principal source of Lgi4 in the developing nerve.

The strikingly similar peripheral nerve phenotype of Adam 22 mutant animals and claw paw animals (Henry et al., 1991; Sagane et al., 2005; Bermingham et al., 2006), together with the observation that Lgil and Lgi4 bind to cells ectopically expressing Adam22 (Fukata et al., 2006; Sagane et al., 2008), suggest that Adam 22 functions as a Lgi4 receptor in nerve development. To test whether binding of Lgi4 to Adam 22 is direct and/or requires the presence of additional molecules at the cell membrane, we used human IgG-Fc fusion proteins that contain the ectodomain of Adam22 (Fig. 2A). The fusion proteins contained either normal Adam 22 or Adam 22 with a D509N (Asp to Asn substitution at position 509) mutation in the disintegrin-like domain that is analogous to the ju160 allele of C. elegans Adm1/Unc-71, which causes axon guidance and sex myoblast migration defects (Huang et al., 2003). Fusion proteins were incubated with conditioned medium of $\mathrm{CHO}$ cells expressing and secreting myc-tagged Lgi4, and precipitated with protein A-Sepharose beads. The precipitated proteins were analyzed by Western blot (Fig. 2B). Adam 22-Fc protein was found to efficiently precipitate Lgi4 from conditioned medium. In contrast, no Lgi4 protein was found precipitated with the Adam $22-\mathrm{Fc}$ protein containing the D509N mutation. These results indicate that binding of Lgi4 to Adam 22 does not require additional membrane associated receptors and that binding depends on the integrin-binding domain of Adam 22 .

Since several other LGI and ADAM family molecules, in addition to Lgi4 and Adam22, are expressed in the PNS (sup- 
plemental Fig. 1, available at www. jneurosci.org as supplemental material), we examined binding of Lgi4, Lgi2 and Lgi3 to cell surface expressed Adam proteins Adam22, Adam23 and Adam11. Conditioned medium of $\mathrm{CHO}$ cells overexpressing tagged versions of Lg4, Lgi2 and Lgi3 were incubated with HeLa cells expressing C-terminal GFP fusions of Adam22, Adam 23 or Adam 11 and binding was detected by immunocytochemistry. Lgi4 binds, in addition to Adam22, also to Adam 11 and Adam23, both of which are expressed in Schwann cells and sensory neurons (Fig. 2C; supplemental Fig. 1, available at www.jneurosci.org as supplemental material). Lgi2 and Lgi3 are also expressed in the peripheral nerve and bind to Adam11 and Adam22/Adam23, respectively (Fig. 2C).

Whether Adam 11 and/or Adam 23 contribute to Lgi4 function as alternative Lgi4 receptors in the peripheral nerve is unclear. If Adam 11 or Adam 23 function as ancillary receptors for Lgi4, the peripheral nerves in Lgi4-deficient mice should be more severely affected than the nerves in Adam 22 mutant mice. When we compared the developmental stage of sciatic nerves derived from wild-type, claw paw (Lgi4 ${ }^{\text {clp } / c l p}$ ) and Adam22-deficient (Adam22 $2^{\text {neo/neo }}$ ) animals at P12, the peripheral nerves in both mutant animals were found to be hypomyelinated. However, the claw paw nerve phenotype appears more severe with fewer myelin figures (Fig. 3A). Immunohistochemical and Western blot analyses confirm the histological analyses and show that more Schwann cells have progressed to the myelinating stage in Adam 22 mutant animals compared with claw paw animals, as evidenced by the higher levels of myelin protein zero (Mpz) and Krox20 protein (Fig. 3C) and the larger number of cells expressing Mpz and Krox20 (Fig. 3B). This less severe Adam22 mutant nerve phenotype suggests that additional Lgi4 receptors exist and can partially compensate for the loss of Adam22.

\section{Cell autonomous function of Adam22}

Adam22 is expressed by neurons as well as Schwann cells (Sagane et al., 2005) (supplemental Fig. 1, available at www.jneurosci.org as supplemental material) suggesting that Lgi4 could function in an autocrine or/and paracrine manner. To determine whether Adam 22 expression is required on Schwann cells, on neurons, or both, we generated primary sensory neuron cultures from Adam $22^{+/ \text {neo }}$ and Adam $22^{\text {neo/neo }}$ embryos and seeded these cultures with wild-type rat Schwann cells. Rat Schwann cells readily ensheath and myelinate Adam $22^{+/ n e o}$ neurons [myelin basic protein-positive (red) myelin sheaths surrounding neurofilamentpositive (green) neurites], whereas Adam $22^{\text {neo/neo }}$ neurons are not myelinated (Fig. 4). These results indicate that Adam 22 function is required in the neuron, but do not exclude an additional function in Schwann cells.

To further explore a possible Schwann cell-autonomous function of Adam 22 and to corroborate its neuronal function in nerve development, we generated a conditional Adam22-null allele (Fig. 5A) and bred it to homozygosity in the presence of the

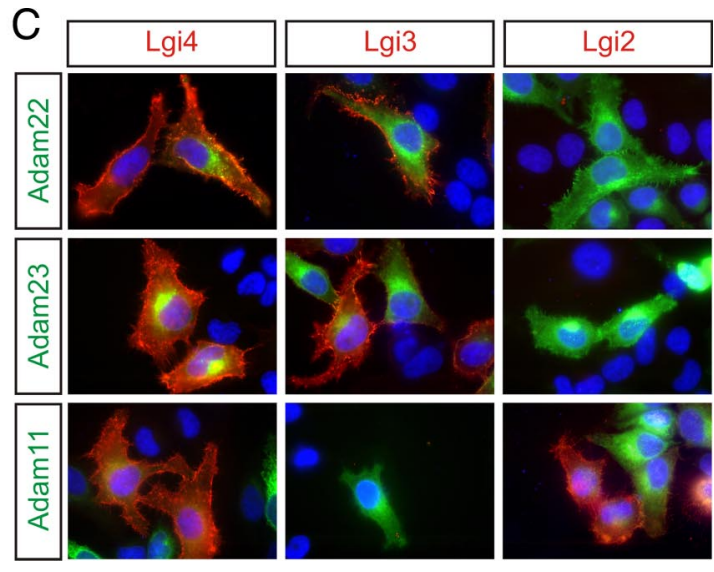

Figure 2. Lgi4 binds to Adam receptors. $A$, Soluble fusion proteins between the ectodomain of Adam22, either wild type or

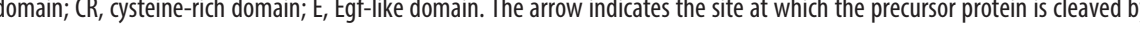
ansfected with a myc-tagged Lgi4 expression cassette (1). The disintegrin mutant Adam 22 Fc-fusion protein (2) does not precipitate Lgi4 from conditioned medium. The precipitated Adam22-Fc fusion proteins were detected using an anti-human $\mathrm{Fc}$ (left panel) while myc-tagged Lgi4 was detected using a myc antibody (right panel). C, Lgi4 (red) binds to the surface of and Adam 11 (-GFP fusion; green). Lgi2 (red) and Lgi3 (red), both of which are expressed in sensory neurons and in Schwann cells (supplemental Fig. 1, available at www.jneurosci.org as supplemental material), bind with different specificity to Adam22, Adam23 and Adam11. Lgi4, Lgi2 and Lgi3 are added to transfected HeLa cells in the form of conditioned media derived from CHO cells overexpressing either myc-tagged Lgi4 (red), or V5 tagged Lgi2 (red) or Lgi3 (red).

Wnt1Cre transgene, thereby deleting Adam 22 in all neural crest derivatives, including sensory neurons, endoneurial fibroblasts and Schwann cells, but not in spinal motor neurons. The sciatic nerve is a mixed nerve containing both primary sensory and motor fibers (see diagram in Fig. 5C). Histological and immunostaining analysis of myelin formation in the sciatic nerve of these mice revealed a mosaic pattern of normally myelinated fibers and promyelin arrested fibers (Fig. $5 B, C$ ). Most of the myelinated axons express choline acetyltransferase (ChAT) (Fig. 5C, top), indicating they are motor fibers. Therefore, motor neurons are myelinated normally by Adam22-deficient Schwann cells. In P12 Wnt1:Adam $22^{\text {Lox/Lox }}$ sciatic nerves, as in Lgi4-deficient claw paw mice of similar age, nonmyelinated fibers are associated with predominantly Oct6-positive promyelinating cells (Fig. 5C, bottom), whereas Krox20-positive Schwann cells are invariably myelinating cells as evidenced by Mpz protein expression (Fig. 5C, middle; see also Fig. 3B). Examination of the ventral motor and dorsal sensory roots revealed normal myelinated motor fibers and severely hypomyelinated sensory fibers, respectively (Fig. $5 B, D)$. These data demonstrate that Adam 22 is required in neurons, whereas deleting Adam 22 in Schwann cells has no effect on myelin formation. The latter conclusion is further illustrated by the normal microscopic appearance of cross sections of sciatic nerves derived from mice in which Adam 22 is deleted, through the action of the DhhCre transgene, in Schwann cells only (Fig. $5 E$ ). Thus, axonally expressed Adam 22 serves as the major receptor for Schwann cell-derived Lgi4 in PNS myelination.

\section{Does Lgi4-Adam 22 interaction modulate Nrg1 signaling?}

How does Lgi4 binding to axonal Adam22 drive Schwann cell myelination? The multitude of axon-Schwann cell signaling and interacting molecules provide numerous possible mechanisms by which Lgi4-Adam 22 may promote myelin formation. One possibility is that Lgi4-Adam 22 promotes myelination through mod- 
A

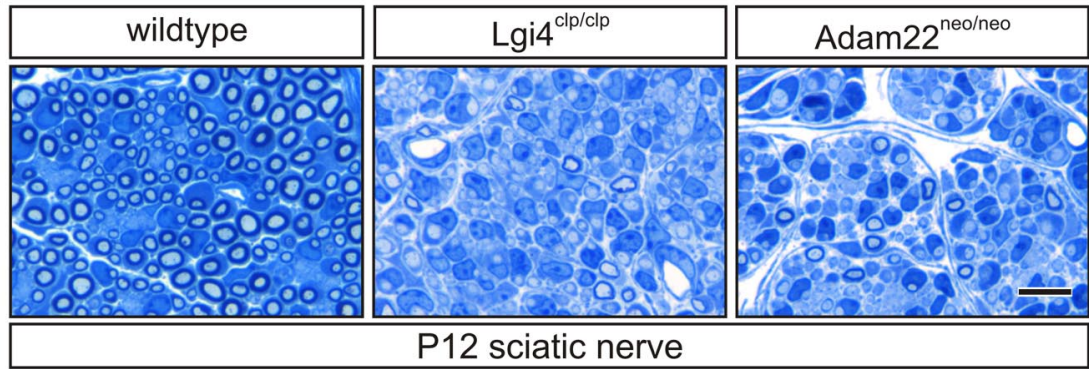

B
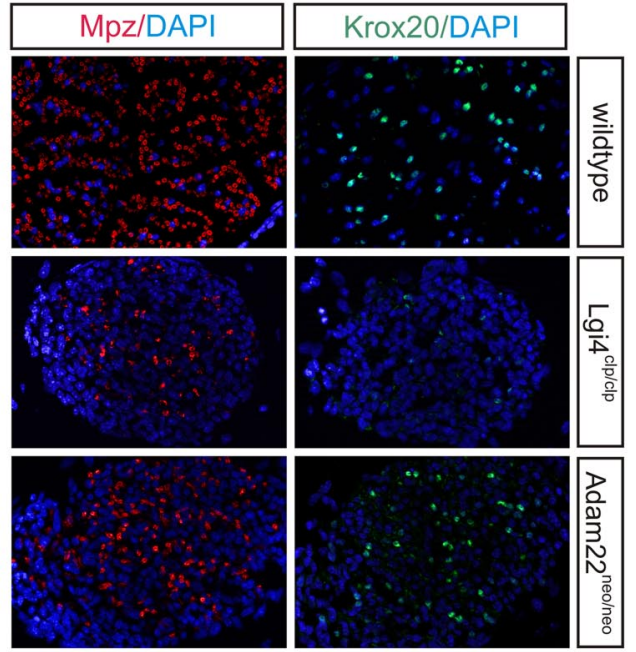

P12 sciatic nerve
C

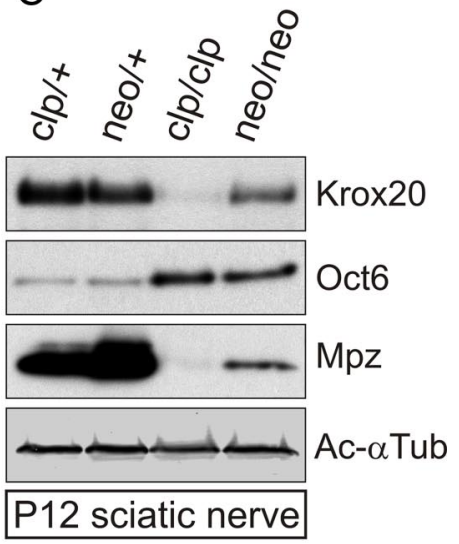

Figure 3. Comparison of sciatic nerves of $L$ gi 4 and Adam22 deficient animals. $A$, Toluidine blue-stained cross sections of sciatic nerve of claw paw ( $\left(\mathrm{gi} \mathrm{i}^{\mathrm{cl} / \mathrm{C} / \mathrm{lP}}\right.$ ) and Adam22 (Adam22 ${ }^{\text {neo/neo }}$ ) mutant show similarities in extent of myelin formation at P12. Representative pictures of 3 individuals per genotype examined. Scale bar, $10 \mu \mathrm{m}$. B, Mpz and Krox20 staining of cross sections of sciatic nerves derived from Claw paw, Adam22 mutant and wild-type animals demonstrate a larger fraction of myelinating Schwann cells (Krox20 and Mpz positive) in Adam22 mutants versus claw paw animals. C, Western blotting corroborates this observation as elevated levels of the promyelinating marker 0ct6 and reduced Mpz expression are observed in both mutant animals. The more advanced stage of Schwann cell differentiation in Adam $22^{\text {neo/neo }}$ versus claw paw animals is again evident from the higher Krox20 and Mpz levels in Adam22 $2^{\text {neo/neo }}$ nerves.

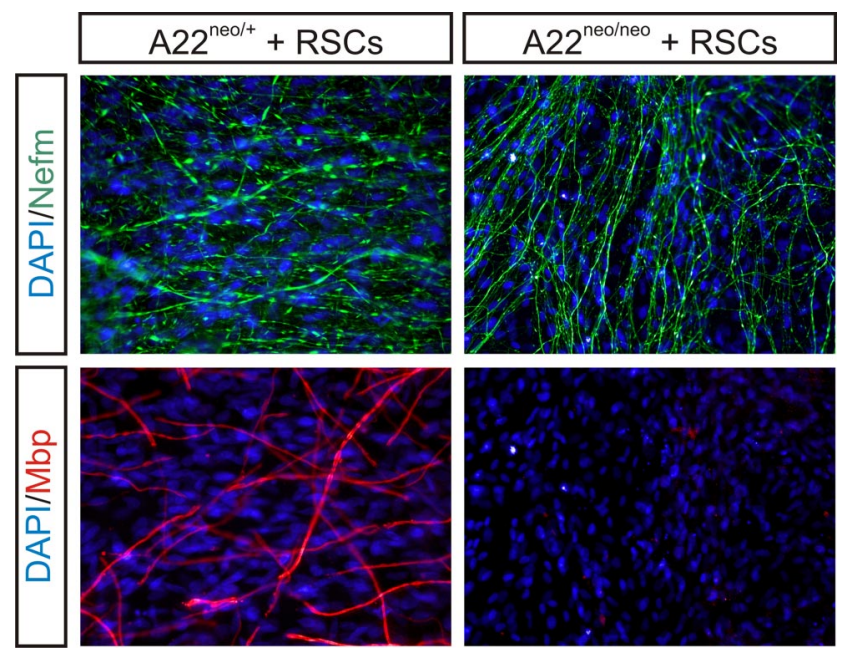

Figure 4. Adam 22 is a neuronal receptor required for myelin formation by Schwann cells. Wild-type rat $S$ chwann cells ensheath and myelinate the neurites of embryonic sensory neurons derived from Adam22 $2^{\text {neo/+ }}$ but not Adam22 $2^{\text {neo/neo }}$ mutant embryos in vitro. The neurites in these cultures are visualized by neurofilament staining (Nefm; green) and myelin is visualized by myelin basic protein (Mbp; red) staining. The nuclei of Schwann cells and neurons are revealed with DAPI (blue) stain. ulating the axonal membrane expression or processing of type III Nrg1. Type III $\mathrm{Nrg} 1$ is required for myelination and the extent of myelination is proportional to the level of type III Nrg1 expression on the axonal membrane (Michailov et al., 2004; Taveggia et al., 2005). To test whether deficient clustering or processing of Nrg1 on Adam 22 deficient axons could account for the hypomyelination phenotype in these mutant mice, we examined the surface expression and signaling potential of type III Nrg1. Heterodimeric fusion proteins of the ErbB2 and ErbB3 extracellular domains with the human Ig Fc domain have been used previously to demonstrate type III Nrg1 expression on axons (Taveggia et al., 2005). Using these fusion proteins, membrane bound Nrg1 could be readily detected on the axons of wild-type as well as Adam 22 mutant primary sensory neurons (Fig. 6A).

We next tested the ability of Nrg1 on wild-type and Adam 22 deficient axons to activate signaling in Schwann cells. Axonal membrane of sensory neurons robustly activate the phosphatidylinositol-3-kinase (PI3K) and extracellular signal-regulated kinase (Erk) pathways in Schwann cells and it has been demonstrated that phosphorylation of AKT, a major target of PI3K, depends on the activity of type III Nrg1 (Ogata et al., 2004; Taveggia et al., 2005). We prepared membranes from sensory neuron cultures derived from wild-type or Adam 22 mutant embryos and seeded these on Schwann cells. Both preparations activate the PI3K and Erk pathways to the same extent (Fig. 6B) in Schwann cells. Thus, these experiments eliminate the simple hypothesis that Lgi4-Adam 22 is required for membrane localization or activity of Nrg1 type III.

\section{Discussion}

Here we show, through selective deletion of the Lgi4 gene in the Schwann cell lineage, that Schwann cells are the principal source of Lgi4 in the developing peripheral nerve tissues. This result corroborates our earlier conclusions from nerve transplantation and siRNA-mediated inhibition of Lgi4 expression in Schwann cells (Darbas et al., 2004; Bermingham et al., 2006). Interestingly, we found that sensory neurons also express Lgi4 at low levels. Either this neuronal Lgi4 is not transported and secreted along the axon or its expression is too low, as deletion of Lgi4 in both Schwann cells, endoneurial fibroblasts and sensory neurons (as in Wnt1-Cre:Lgi4 ${ }^{\text {Lox/Lox }}$ animals) results in a similar hypomyelinating nerve phenotype as observed in DhhCre:Lgi4 ${ }^{\text {Lox/Lox }}$ animals in which Lgi4 is only deleted in Schwann cells and endoneurial fibroblasts. Also, we found that Lgi2 and Lgi3 are expressed at low level in Schwann cells and at higher levels in sensory neurons (Bermingham et al., 2006). Apparently, Lgi2 and Lgi3 proteins do not substitute for the loss of Lgi4 in claw paw animals. In line with this we found that Lgi2 does not bind to Adam 22 at all. However, Lgi3 does bind to Adam22, suggesting that Lgi3 is not secreted at sufficient levels at the right developmental time, or that Lgi4 and 


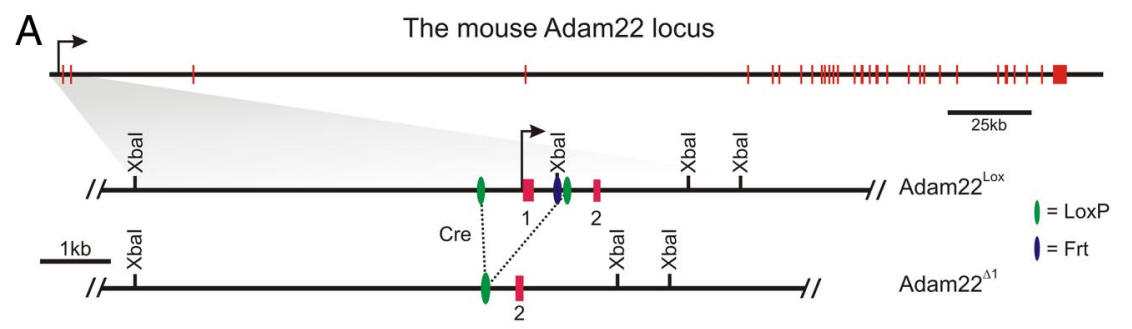

B

a
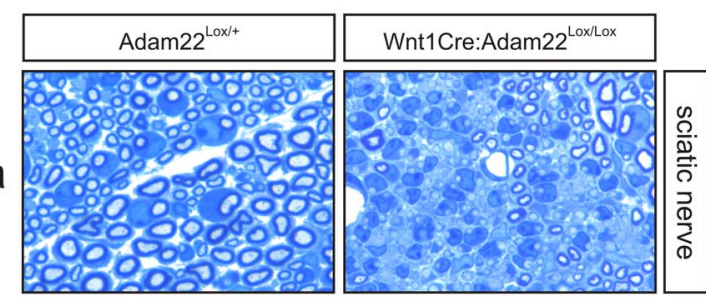

b
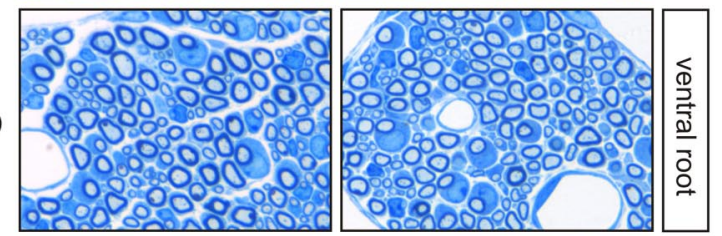

C

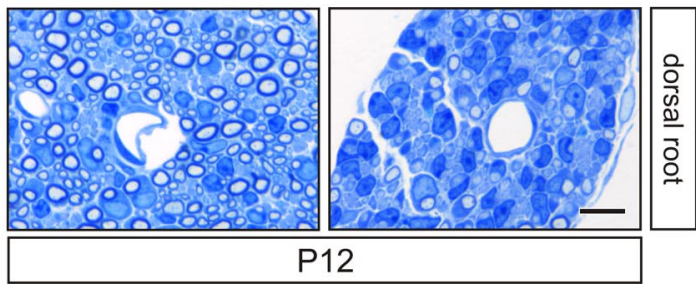

C
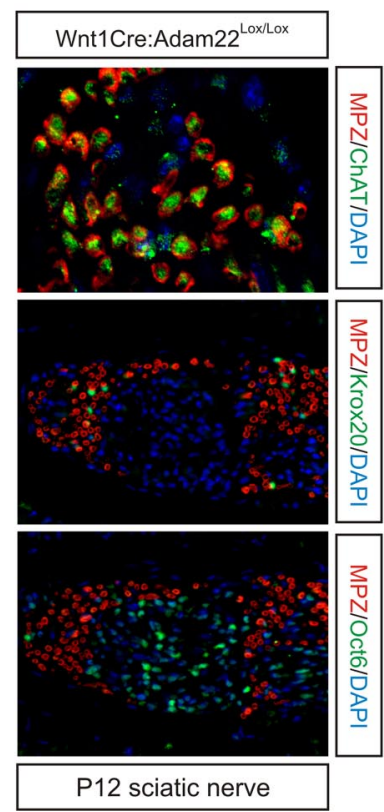

D

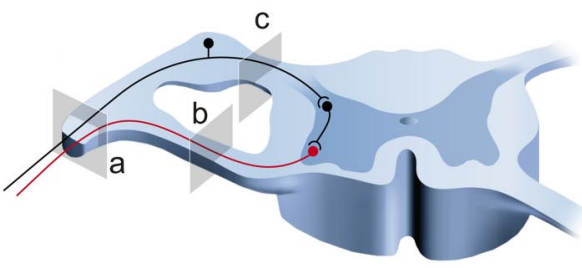

$E$
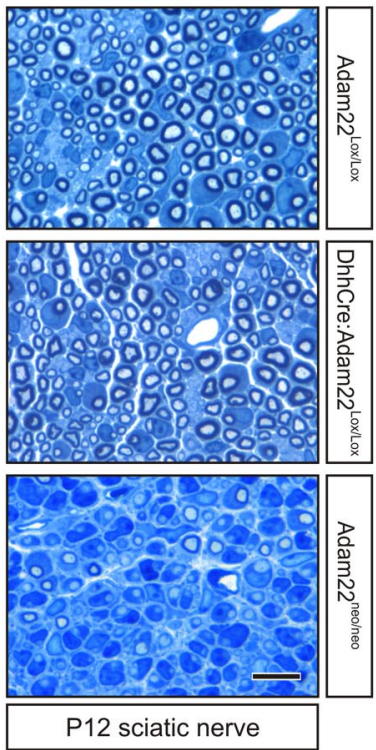

Figure 5. Neuron cell-autonomous function of Adam22. A, Schematic depiction of the mouse Adam22 locus, the conditional allele (Adam22 $2^{\text {Lox }}$ ), and the null allele (Adam22 ${ }^{\Delta t}$ ) that results from the action of the Cre recombinase. The Adam22 gene consists of $>30$ exons (red vertical bars on top line) that span $\sim 300 \mathrm{~kb}$ of genomic sequences on mouse chromosome 5 . The promoter is indicated with an arrow. B, Conditional deletion of Adam22 in neural crest derivatives using the Wnt 1 Cre driver. Toluidine blue staining of sections of nerves from Adam22 ${ }^{\text {Lox/+ }}$ and Wnt $1 \mathrm{Cre}$ : Adam22 $2^{\text {Lox } / \text { Lox }}$ mice at three different anatomical levels $\boldsymbol{a}-\boldsymbol{c}($ scale bar, $10 \mu \mathrm{m})$. These levels are schematically depicted in $\boldsymbol{D}$. Motor fibers are indicated in red and sensory fibers in black. C, Immunostaining for ChAT (green) and Mpz (myelin; red) shows that most myelinated axons are positive for ChAT. Schwann cells in the nonmyelinated areas of the nerve are Krox20 negative and Oct6 positive. E, Myelination of nerve fibers in the sciatic nerve of mice homozygous for the Adam22 conditional null allele (Adam22 ${ }^{\text {Lox Lox }}$ ) appears normal and is comparable to that observed in sciatic nerve of DhhCre:Adam22 ${ }^{\text {Lox/Lox }}$ mice. In the latter, Adam22 is deleted selectively in Schwann cells. Complete deletion of Adam22 (Adam22 ${ }^{\text {neo/neo }}$ ) results in a hypomyelinating phenotype. The scale bar represents $10 \mu \mathrm{m}$.

A
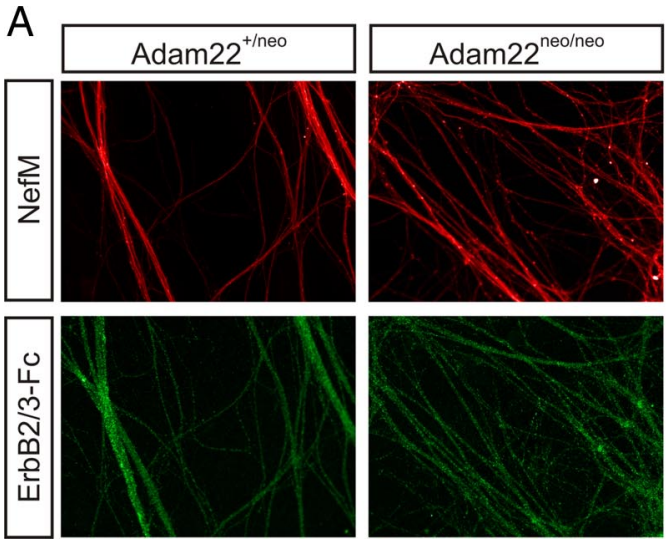
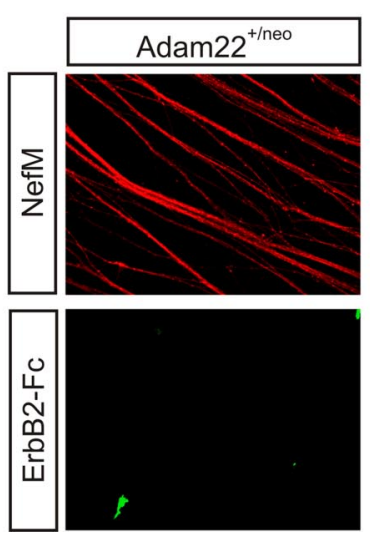

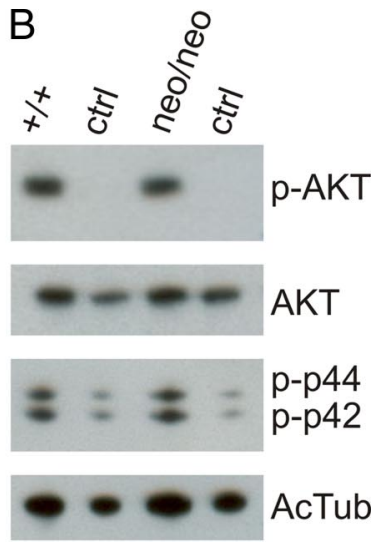

Figure 6. Adam 22 and contact-dependent Nrg1 signaling. $A$, Embryonic sensory neuron cultures derived from $A d a m 22^{\text {neo/+ }}$ ( 9 cultures derived from 5 embryos; $9 / 9$ positive for ErbB2/3 binding) and Adam22 $2^{\text {neo/neo }}$ ( 6 cultures from 3 embryos; $6 / 6$ positive for ErbB2/3 binding) mutant embryos express typelll Nrg1 on their neurites as evidenced by binding of the ErbB2/3-Fc fusion proteins. Neurites are visualized with neurofilament (NefM) antibodies (red) and the $\mathrm{Fc}$ moiety of the fusion proteins is visualized with an anti-human $\mathrm{Fc}$ antibody (green). ErbB2-Fc does not bind Nrg1 and serves here as a control. Magnification $20 \times . \boldsymbol{B}$, Axonal membranes were prepared from Adam22 $2^{+/+}\left(+/+\right.$) and Adam22 ${ }^{\text {neo/neo }}$ (neo/neo) primary sensory neuron cultures and centrifuged onto Schwann cells. After 30 min lysates were probed with antibodies for phosphorylated-Akt (p-AKT), total Akt (AKT), and for phosphorylated Erks (p-p42 and p-p44). A lysate of Schwann cells exposed to fresh serum-free medium served as a control (ctrl). The experiment was performed twice, in duplicate. 
Lgi3 are qualitatively different, perhaps reflecting differential interactions through their LRR domains. Thus, we find that Lgi4 is the major LGI protein required for Schwann cell differentiation and myelination.

Lgi4 appears to function in PNS myelination principally through interaction with the transmembrane protein Adam 22 as Lgi4 binds directly to the extracellular domain of Adam 22 and deletion of Adam 22 in mice results in a peripheral nerve phenotype that is very similar to that observed in claw paw animals (Sagane et al., 2005) (Fig. 3). Adam22 belongs to a small subfamily of ADAM proteins, including Adam23 and Adam11, which are predominantly expressed in the nervous system (Yang et al., 2006). Adam 22, like Adam 23 and Adam 11, lacks metalloproteinase activity, and therefore these ADAM proteins have been suggested to mainly function as integrin receptors through their disintegrin domain (Yang et al., 2006). Soluble forms of the Adam22 extracellular domain inhibit cell proliferation in an integrin-dependent manner (D'Abaco et al., 2006). Interestingly, it was found that reexpression of Lgil in the glioma cell line T98G (the glioma cell line in which Lgil originally was found inactivated; hence the name Leucine-rich Glioma Inactivated) reduces proliferation and cell migration (Chernova et al., 1998; Kunapuli et al., 2003). It is unclear whether these observations are relevant for the role of Adam 22 in the peripheral nervous system. It is possible that Lgi4 binding to Adam 22 modulates (either positively or negatively) Adam22-integrin interactions, as Lgi4 binds to the disintegrin-like domain of Adam22. Indeed, Adam22 interacts with a number of integrins that are expressed on Schwann cells (in particular $\alpha 6 \beta 1$ and $\alpha 6 \beta 4$ ) and are involved in axonal sorting and myelin stabilization (Berti et al., 2006; D'Abaco et al., 2006). However, the actions of these integrins have been interpreted in terms of their interaction with the Schwann cell extracellular matrix and not with the axon. It is tempting to speculate that Lgi4 regulates interactions between axonal Adam 22 and Schwann cell integrins to allow progression of the Schwann cell's inner mesaxon over the axonal membrane and initiate myelin formation, a process that is inhibited in the absence of Lgi4 or Adam 22.

An alternative mechanism of Lgi4-Adam22 function is suggested by work of Fukata and colleagues (Fukata et al., 2006). Adam 22 was found associated with Lgil when both Adam 22 and Lgil coprecipitated with Stargazin and PSD95 from rat brain membrane preparations. Lgil, which is found frequently mutated in autosomal dominant partial epilepsy with auditory features (ADPEAF) (for review, see Nobile et al., 2009), enhances AMPA-receptor-mediated synaptic transmission. It was suggested that Lgil binding to Adam22 stabilizes the Stargazin/ AMPAR/PSD95/Adam22 complex in the postsynaptic membrane. Similarly, it is possible that Lgi4 binding to axonal Adam22 stabilizes complexes at the axonal surface that are involved in Schwann cell mesaxon progression and myelination. We have begun to explore this possibility here by examining the surface expression and signaling potential of membrane bound type III NRG1, a known regulator of myelination. We found that NRG1 is expressed on the axonal membrane of Adam $22^{\text {neo/neo }}$ neurons and that these membranes activate PI3K and Erk as effectively as membranes derived from wild-type neurons, suggesting that Lgi4-Adam 22 does not function by modulating Nrg1-ErbB2/3 interactions.

To further explore the mechanism of Adam22 function in myelination it will be important to identify proteins that bind to the cytoplasmic tail of Adam22, which includes a PDZ binding motif. Interestingly, it was recently demonstrated that accumula- tion of the MAGUKs PSD93/PSD95 at the juxtaparanodal membrane of myelinated axons depends on Adam22 (Ogawa et al., 2010). It is unlikely that these interactions are important for the earlier function of Adam22 as PSD93/PSD95 double knock out animals, though severely affected, form myelin (Horresh et al., 2008).

In conclusion, by identifying Adam 22 as the major axonal receptor for the Schwann cell-derived factor Lgi4, we have revealed a new molecular component in Schwann cell-neuron interactions that govern myelin formation in the PNS. In contrast to previously identified ligand-receptor pairs that function in PNS development, Lgi4 is secreted from Schwann cells and binds to axonally expressed Adam22. How Lgi4 and Adam22 interact with other axon-Schwann cell signaling molecules is an important unanswered question, the answer to which may provide novel approaches for treating myelin disease.

\section{References}

Archelos JJ, Roggenbuck K, Schneider-Schaulies J, Linington C, Toyka KV, Hartung HP (1993) Production and characterization of monoclonal antibodies to the extracellular domain of P0. J Neurosci Res 35:46-53.

Bermingham JR Jr, Shearin H, Pennington J, O’Moore J, Jaegle M, Driegen S, van Zon A, Darbas A, Ozkaynak E, Ryu EJ, Milbrandt J, Meijer D (2006) The claw paw mutation reveals a role for Lgi4 in peripheral nerve development. Nat Neurosci 9:76-84.

Berti C, Nodari A, Wrabetz L, Feltri ML (2006) Role of integrins in peripheral nerves and hereditary neuropathies. Neuromolecular Med 8: 191-204.

Birchmeier C, Nave KA (2008) Neuregulin-1, a key axonal signal that drives Schwann cell growth and differentiation. Glia 56:1491-1497.

Brockes JP, Fields KL, Raff MC (1979) Studies on cultured rat Schwann cells. I. Establishment of purified populations from cultures of peripheral nerve. Brain Res 165:105-118.

Chan JR, Cosgaya JM, Wu YJ, Shooter EM (2001) Neurotrophins are key mediators of the myelination program in the peripheral nervous system. Proc Natl Acad Sci U S A 98:14661-14668.

Chernova OB, Somerville RP, Cowell JK (1998) A novel gene, LGI1, from $10 \mathrm{q} 24$ is rearranged and downregulated in malignant brain tumors. Oncogene 17:2873-2881.

D’Abaco GM, Ng K, Paradiso L, Godde NJ, Kaye A, Novak U (2006) ADAM22, expressed in normal brain but not in high-grade gliomas, inhibits cellular proliferation via the disintegrin domain. Neurosurgery 58 : 179-186; discussion 179-186

Danielian PS, Muccino D, Rowitch DH, Michael SK, McMahon AP (1998) Modification of gene activity in mouse embryos in utero by a tamoxifeninducible form of Cre recombinase. Curr Biol 8:1323-1326.

Darbas A, Jaegle M, Walbeehm E, van den Burg H, Driegen S, Broos L, Uyl M, Visser P, Grosveld F, Meijer D (2004) Cell autonomy of the mouse claw paw mutation. Dev Biol 272:470-482.

Estable-Puig JF, Bauer WC, Blumberg JM (1965) Technical note; paraphenylenediamine staining of osmium-fixed plastic embedded tissue for light and phase microscopy. J Neuropathol Exp Neurol 24:531-536.

Farley FW, Soriano P, Steffen LS, and Dymecki SM (2000) Widespread recombinase expression using FLPeR (flipper) mice. Genesis 28:106-110.

Fukata Y, Adesnik H, Iwanaga T, Bredt DS, Nicoll RA, Fukata M (2006) Epilepsy-related ligand/receptor complex LGI1 and ADAM22 regulate synaptic transmission. Science 313:1792-1795.

Ghazvini M, Mandemakers W, Jaegle M, Piirsoo M, Driegen S, Koutsourakis M, Smit X, Grosveld F, Meijer D (2002) A cell type-specific allele of the POU gene Oct- 6 reveals Schwann cell autonomous function in nerve development and regeneration. EMBO J 21:4612-4620.

Henry EW, Eicher EM, Sidman RL (1991) The mouse mutation claw paw: forelimb deformity and delayed myelination throughout the peripheral nervous system. J Hered 82:287-294

Horresh I, Poliak S, Grant S, Bredt D, Rasband MN, Peles E (2008) Multiple molecular interactions determine the clustering of Caspr2 and Kv1 channels in myelinated axons. J Neurosci 28:14213-14222.

Huang X, Huang P, Robinson MK, Stern MJ, Jin Y (2003) UNC-71, a disintegrin and metalloprotease (ADAM) protein, regulates motor axon 
guidance and sex myoblast migration in C. elegans. Development 130:3147-3161.

Jaegle M, Ghazvini M, Mandemakers W, Piirsoo M, Driegen S, Levavasseur F, Raghoenath S, Grosveld F, Meijer D (2003) The POU proteins Brn-2 and Oct-6 share important functions in Schwann cell development. Genes Dev 17:1380-1391.

Jessen KR, Mirsky R (2005) The origin and development of glial cells in peripheral nerves. Nat Rev Neurosci 6:671-682.

Joseph NM, Mukouyama YS, Mosher JT, Jaegle M, Crone SA, Dormand EL, Lee KF, Meijer D, Anderson DJ, Morrison SJ (2004) Neural crest stem cells undergo multilineage differentiation in developing peripheral nerves to generate endoneurial fibroblasts in addition to Schwann cells. Development 131:5599-5612.

Kunapuli P, Chitta KS, Cowell JK (2003) Suppression of the cell proliferation and invasion phenotypes in glioma cells by the LGI1 gene. Oncogene 22:3985-3991.

Maurel P, Einheber S, Galinska J, Thaker P, Lam I, Rubin MB, Scherer SS, Murakami Y, Gutmann DH, Salzer JL (2007) Nectin-like proteins mediate axon Schwann cell interactions along the internode and are essential for myelination. J Cell Biol 178:861-874.

Michailov GV, Sereda MW, Brinkmann BG, Fischer TM, Haug B, Birchmeier C, Role L, Lai C, Schwab MH, Nave KA (2004) Axonal neuregulin-1 regulates myelin sheath thickness. Science 304:700-703.

Monk KR, Naylor SG, Glenn TD, Mercurio S, Perlin JR, Dominguez C, Moens CB, Talbot WS (2009) A G protein-coupled receptor is essential for Schwann cells to initiate myelination. Science 325:1402-1405.

Nave KA, Salzer JL (2006) Axonal regulation of myelination by neuregulin 1. Curr Opin Neurobiol 16:492-500.

Nobile C, Michelucci R, Andreazza S, Pasini E, Tosatto SC, Striano P (2009) LGI1 mutations in autosomal dominant and sporadic lateral temporal epilepsy. Hum Mutat 30:530-536.

Ogata T, Iijima S, Hoshikawa S, Miura T, Yamamoto S, Oda H, Nakamura K,
Tanaka S (2004) Opposing extracellular signal-regulated kinase and Akt pathways control Schwann cell myelination. J Neurosci 24:6724-6732.

Ogata T, Yamamoto S, Nakamura K, Tanaka S (2006) Signaling axis in Schwann cell proliferation and differentiation. Mol Neurobiol 33:51-62.

Ogawa Y, Oses-Prieto J, Kim MY, Horresh I, Peles E, Burlingame AL, Trimmer JS, Meijer D, Rasband MN (2010) ADAM22, a Kv1 channel-interacting protein, recruits membrane-associated guanylate kinases to juxtaparanodes of myelinated axons. J Neurosci 30:1038-1048.

Sagane K, Hayakawa K, Kai J, Hirohashi T, Takahashi E, Miyamoto N, Ino M, Oki T, Yamazaki K, Nagasu T (2005) Ataxia and peripheral nerve hypomyelination in ADAM22-deficient mice. BMC Neurosci 6:33.

Sagane K, Ishihama Y, Sugimoto H (2008) LGI1 and LGI4 bind to ADAM22, ADAM23 and ADAM11. Int J Biol Sci 4:387-396.

Schulte U, Thumfart JO, Klöcker N, Sailer CA, Bildl W, Biniossek M, Dehn D, Deller T, Eble S, Abbass K, Wangler T, Knaus HG, Fakler B (2006) The epilepsy-linked Lgil protein assembles into presynaptic Kvl channels and inhibits inactivation by Kvbeta1. Neuron 49:697-706.

Seals DF, Courtneidge SA (2003) The ADAMs family of metalloproteases: multidomain proteins with multiple functions. Genes Dev 17:7-30.

Spiegel I, Adamsky K, Eshed Y, Milo R, Sabanay H, Sarig-Nadir O, Horresh I, Scherer SS, Rasband MN, Peles E (2007) A central role for Necl4 (SynCAM4) in Schwann cell-axon interaction and myelination. Nat Neurosci 10:861-869.

Sun YP, Deng KJ, Wang F, Zhang J, Huang X, Qiao S, Zhao S (2004) Two novel isoforms of Adam 23 expressed in the developmental process of mouse and human brains. Gene 325:171-178.

Taveggia C, Zanazzi G, Petrylak A, Yano H, Rosenbluth J, Einheber S, Xu X, Esper RM, Loeb JA, Shrager P, Chao MV, Falls DL, Role L, Salzer JL (2005) Neuregulin-1 type III determines the ensheathment fate of axons. Neuron 47:681-694.

Yang P, Baker KA, Hagg T (2006) The ADAMs family: coordinators of nervous system development, plasticity and repair. Prog Neurobiol 79:73-94. 